\title{
The Conceptual Project in Child Variance ${ }^{1}$
}

\author{
Sabin Head ${ }^{2}$ and William C. Rhodes \\ University of Michigan
}

This report covers highlights from a survey of the literature on emotional disturbance. The survey was begun in 1970 by the Conceptual Project in Child Variance at the University of Michigan. The Project has developed the survey to serve both as a model for graduate student training and as a source of materials for graduate level programs across the United States. Results are being published in a four-volume work, A Study of Child Variance, which examines theories, intervention practices, and service delivery systems, and presents an overview with a look toward the future. The activities of the graduate students and of professional workshop groups are described, and a summary of Project materials is also reported.

The Conceptual Project in Child Variance has been in existence at the University of Michigan since 1970. It is a Special Project funded by the Bureau of the Education for the Handicapped under the U.S. Office of Education. The Project's primary purpose is to survey the vast but scattered literature bearing on emotional disturbance, with the goal of producing materials suitable for graduate training programs in the teaching of the emotionally disturbed. A second purpose is to serve as a prototype for the training of graduate students. A third purpose is to actively disseminate the research results to an audience of teacher trainers and state personnel representing, in principle, each graduate level program in the education of the emotionally disturbed in the United States and each state education department.

Manuscript received in final form November 20, 1973

${ }^{1}$ This study was prepared under grants OEG-0-70-4806(603), 0EG-0-71-3680(603), and 0EG-0-72-3680(603) from the Bureau for Education of the Handicapped, U.S. Office of Education. The project is located in the Institute for the Study of Mental Retardation and Related Disabilities at the University of Michigan, Ann Arbor. This support is gratefully acknowledged.

${ }^{2}$ Requests for reprints should be sent to Sabin Head, Institute for the Study of Mental Retardation and Related Disabilities, University of Michigan, 130 South First Street, Ann Arbor, Michigan 48108. 
The intended effect of the Project, in its simplest form, is to broaden the scope of materials and concepts available for an integrated multidisciplinary approach to the training of professionals to deal with variant children. It is also intended that materials be a useful resource to professionals by systematically covering widely differing alternative theories, techniques, and institutions focused on variant children.

Though there is an insistent demand for the invention of more and better theories and practices, the Project has found no paucity of them already extant in the literature. Rather the reverse, the welter of complementary and conflicting studies and viewpoints is confusing; what seems needed is a more intense effort at synthesis and more routine communication and cooperation between theorists, trainers, practitioners, and administrators.

\section{RESEARCH STAGES}

In an effort at comprehensiveness, the Project's research has been broken into several stages, and the main results are being published as a series of separate volumes under the title $A$ Study of Child Variance. Supplementary materials

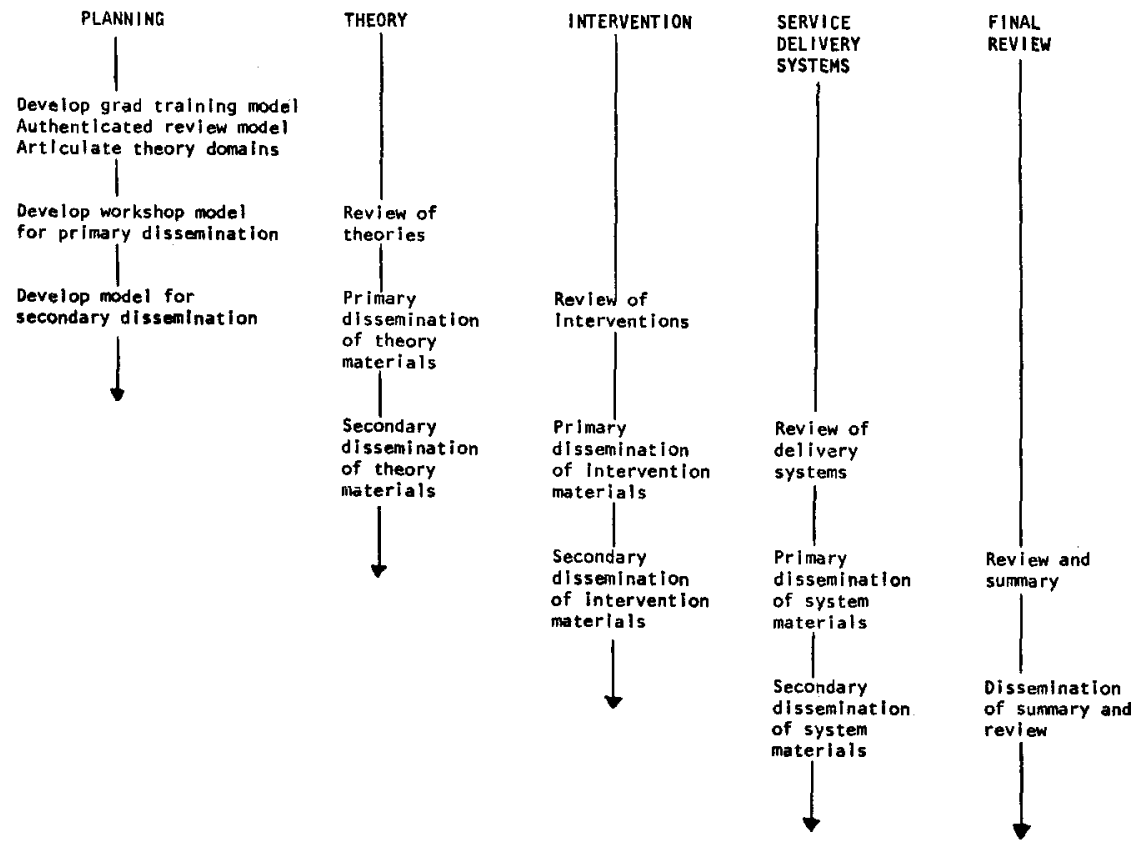

Fig. 1. Timeline of project stages. 
including videotapes and annotated bibliographies are also being published in separate groups corresponding to the stages of research. The timeline of the Project's research efforts is shown in Figure 1.

At the time of this writing, the research on theories has been completed and published as volume I of $A$ Study of Child Variance. The written materials have been published as a book and the supplementary materials are available through the Project. The second stage of research, covering intervention practices, also has been completed and is in press as volume II; preprints of this volume have been circulated. Most of the ancillary materials are available through the Project. In the third stage, covering the service delivery systems, research relating to the first two substages (history and current structure) is being revised preparatory to publication as volume III, and the supplementary materials are being produced. The third substage, documenting the actual functioning of the various institutions as they process variant children in a particular selected community, has just begun. A fourth stage of research is planned to overview the previous research stages with a focus on the future of the treatment of variant children. Its results are projected as the fourth and final volume of the series.

\section{HIGHLIGHTS FROM THE RESEARCH}

The Project's three research stages have proved to be in different worlds, more different than was originally expected. The world of theory is simplified and relatively "pure." Its concerns are abstract and systematic thought at a relatively high level of generality. Though much theory is generated from the bottom up, out of experience, much of it is also generated from the top down, from consideration of primary principles and assumptions. By contrast, the intervention world is a pragmatic one. In particular, it has to deal with the real world of clients and patients. Pure examples of theoretical types are rare in the world of clients, and often clients will partly resemble many different theoretical problems. The problems faced in the world of intervention are not invented; they walk in the front door in all their complexity. In the world of theory, the operating constraints have to do with model formulation, validity, internal consistency, and, to a great extent, simplification. This is as it should be. In the world of intervention, the operating constraints have to do with the nature of the client's complex real world, and with the need to provide effective service. This is also as it should be. The world of delivery systems is not a simple amalgam of the previous two, but brings in another set of complex influences all its own. The institutions do not only embody a particular theoretical viewpoint, nor do they only provide services to clients in need. They embed both in a complex web of ongoing cultural needs; they reflect the culture's priorities as 
well as its problems and its technology to deal with them; they exist to use resources, both of facilities and of personnel; they are deeply involved in the personal lives of their "normal" employees; and they are deeply involved in.the culture's own perception of itself and in the politics by which it runs. Thus, the delivery institutions add a new world of influences, those operating on a system or cultural level. All this, too, is as it should be.

The three worlds of theory, intervention, and service delivery represent a way of dividing labor into thinking and doing and embedding both into the web of society and of history. The unfortunate aspect of this division of labor is that it has led to the establishment of separate houses, between which communication has become narrowly channeled and formalized so that it resembles ambassadorial exchanges.

\section{Theory Highlights}

One of the early realizations in the Project's research on theories of child variance was that, in current philosophy of science terms, these conceptual frameworks are better described as models than as theories. Well formulated theories are stated as testable hypotheses, while models are more informal representations for the purposes of problem solving. The ultimate concern of theories is with some kind of "truth," while the ultimate concern with models is their usefulness. Most "theories" of child variance are models.

A second realization was that the major models of child variance differ in their basic viewpoints or in the aspect of child variance that they focus on. One fundamental difference lies in whether the observed variance is assumed to be a property of the individual or a property of a group role that the individual either plays or has attributed to him.

Another fundamental distinction between the theories is on how child variance is seen. There are three basic paradigms: disability, deviance, and alienation. In the disability paradigm, the variant child is seen as having some incapacity, or some undeveloped capacity, that differentiates him from "normal" children. Normality from this point of view is a prescriptive, idealistic normality. In the deviance paradigm, the variant child is seen as deviating from cultural norms for some reason other than his inability to meet the norms. Here, "normality" is a descriptive, statistical concept. In the alienation paradigm, the individual is seen as declaring his own intentional deviation from cultural norms. The first two paradigms are one-way; they are an extrusion of the individual from the cultural group; the third paradigm is, by contrast, a mutual divorce in which the individual rejects the group as much as he is rejected by it.

Another distinction between the various theories is in the role "unobservables" (such as the ego or the unconscious) are allowed to play in fundamental formulations. Some theories take a positive stand against allowing unob- 
servables to function as primary facts or primary explanatory principles, reserving that role for directly observable events and behaviors. Conceptually, of course, there is no difficulty in handling unobservables; even the "hardest" sciences have an honorable position for them (e.g., force and mass in physics). The chief difference is more a pragmatic issue at base, involving the complexity of the operationalizations needed to characterize the different variables referred to.

Overall, the Project's researchers in theory have demonstrated that the different major models are not in essential conflict because the overlap of their concerns is not that large. They represent multiple, complementary approaches to a multifaceted and vaguely bounded set of problems. A sense that arises from an overview of the available theories is that the set of explanations they provide is not yet complete, not yet rich enough. Countertheorists in general are found to agree with this.

\section{Intervention Highlights}

One of the earliest discoveries in the Project's research on interventions was that the connection between theories and interventions is not hard and fast but is tenuous. Two practitioners who agree in theory may apply different specific tactics to a particular case, while two practitioners with different theoretical viewpoints may use much the same tactics. The relation between theories and interventions is like the relation between an architect's design and the construction materials used. From this point of view, the complementary nature of the essential contributions of both to the outcome becomes important. The design by itself is impotent, while the undesigned application of construction materials is likely to be unimaginative and inefficient.

As with theories, interventions differ in whether they are focused on adapting the individual to the group or on building (or finding) a group context in which the individual is no longer a "problem." Interventions also differ in specifying a referent group. For some, the intent is to match the individual and his natural group; for others, the intent is to match the individual and some "ideal" group - in some forms of the latter, the deviant individual is expected to conform to more adult standards than are represented by his peer group.

Eclecticism seems to be a more prominent feature of interventions and practitioners than of models and theorists. This may reflect the difference between the worlds of theory and interventions, where intervention cannot afford to be as "pure" in the face of many multiproblem cases.

Another distinction between interventions is in their intended effect on the individual. Some seek to help the individual attain some minimum standards of personal achievement, while others seek to assist the individual in realizing his individual potential. Some accept the collective society and provide a vehicle for 
socializing the individual; others reject the current society and aim to build in the individual the means of revolutionizing it, quietly or violently.

As a factor bridging the gap between theories on the one hand and the public service-delivery systems on the other, intervention techniques are often expected to do the impossible. With treatment techniques and diagnostic categories derived, on the whole, from the relatively pure abstractions of theory, the practitioner is asked to intervene constructively into the lives of those society sends to him. The supply of cases is rarely divisible into neat, clean diagnostic types, but is more often a mixed bag of "non-fits," to borrow from Fritz Perls. Society's "fits" are those who produce, consume, trade, and service goods and materials and those who maintain them. The "non-fits" include the lunatics, criminals, dropouts, unemployed, and sick. In contrast to the fits, the non-fits are a highly disorganized subculture and are not financially independent. Practitioners who serve the non-fits, through necessarily inadequate typologies and treatments, are an intermediate class by themselves and include police, welfare workers, hospital and prison staffs, ministers, doctors, psychologists, and the other caretakers of the non-fits.

\section{Service-Delivery System Highlights}

"Service delivery" forces both theories and intervention techniques to face the social world, to adapt to the cultural and political realities of the society and the community. It is in service delivery that theories collide with the intractability of existing social institutions and the personal motivations of men. In form and structure, the social institutions override the intention of theory and the extension of technique, and form them to fit the outline of the historically accrued system. Woven into these palpable systems is the concrete residue of hopes, fears, and aspirations of the past which has been handed on to the future. These collective hopes, fears, and aspirations have crystallized, like stalactites and stalagmites, in the overall mass of the system, to capture newly entering theory and intervention technique in their preordained structure.

There is a preponderance of data in the history of these systems to suggest that service is moved more by the energy of fear and threat of differences than by the energy of compassion or care for those who break or remain outside the prevailing pattern of custom and behavior. If this analysis of the energy behind the system is correct, then a major task in systems change is to dispel the fear of differences which so intensely shapes the form of services. Even if it is not correct, modern society must face up to the extent to which deep human motivations bind and energize the system. Until we deal with these collective motivations, so deeply submerged in the residue of history, we will have great difficulty in remodeling the service institutions that mediate between the culture bearers and the pattern breakers, between the fits and the non-fits. 
It seems that every public service-delivery system, from compulsory education to compulsory incarceration, carries this legacy of the intentions and fears of the past into the institutions of the present. Agents of change will have difficulty in escaping that history. A simple reading of newspaper stories over the past hundred years might show the "repetition compulsion," both of periodic reform efforts and of system maintenance despite these efforts. It may be necessary for change agents to step outside existing institutions to escape the gravitational pull of their history.

It is ironic that the complex of service systems, from education, to corrections, to welfare, to health and mental health, tries so hard to bond the individual to the collective; and yet, so often today, the systems succeed only in intensifying the alienation of many of those they try to help. Perhaps, as is being suggested by social critics like Ivan Illich, we have at last come to the end of school, medicine, corrections, and welfare. No tinkering with the structure, no increment of resources, no perfection of technique can influence the individual and social effects accrued from these systems. If this is so, then one must look to their social alternatives. Societies will always educate, treat, punish, and support their collective members.

Many advocates of change excoriate the economic processes behind these services and suggest that their reform is dependent on basic economic and political change. It is true that services in the United States are directly tied to the economic problems of the society and are direct extensions of the executive branch of government. A look at Scandinavian or Soviet systems, however, suggests that something more than politics and economics is involved in the ills of the systems. Cultural and motivational change, as well as resistance to change, seems to hold a place equal to political and social change.

A major question that comes out of this review of theory, intervention, and service delivery for variant children is, Does it really make a difference to be thoroughly acquainted with this information? As with all knowledge, the answer depends on the user and his uses of such knowledge. The times are certainly ripe for such assimilation and rumination. The storm warnings are rising. A confrontation seems inherent in the directions of the "new culture" and the old around the social treatment of individual variance and deviation. A mastery of the history of our phenomenological and operational treatment of child variance can be used by both groups to map the future.

\section{SUBACTIVITIES OF THE PROJECT}

In addition to its role in reviewing and organizing the conceptual material dealing with child variance, the Project serves two other important functions. It has a specific role in graduate training and in dissemination to an audience of in-service personnel. 


\section{Graduate Training Model}

Almost all the Project's materials are researched and written by graduate students. The Project has involved these graduate student research assistants as the core around which the Project is centered. They represent a variety of disciplines, including anthropology, history psychology, education, law, and medicine. They are given a great deal of autonomy and responsibility, with supervision, in their research, and they receive full authorship credit for their products. Research assistants employed half-time during the academic year, and their respective departments are asked to regard their posts as supervised field or research placements. The students also participate in an ongoing seminar led by the Project Director in which topics of interest to the main research are explored in depth. Their various departments are asked to accept the seminar's academic credit hours toward their degrees. Research papers have often been used by the students to fulfill various program requirements for independent research or review projects, for preliminary exams, and as parts of dissertations.

The seminar itself, with its interdisciplinary focus on a single problem, is an important stimulus to the students' experience. The students are also required to establish contacts with local and national experts in their topic of research for advice, suggestions, and consultation. In addition, each paper is sent through an authentication process (see Figure 2) that involves a critical outside review, a consultation with the reviewer, and subsequent revision of the paper before further circulation. The student later interviews the reviewer on the reviewer's own interests and programs; and finally, the reviewer is brought to Ann Arbor to participate in an experts' conference with the other reviewers. (Both the interview and the conference are videotaped and edited for inclusion in the supplementary materials.) This wealth of outside contacts has proved to be a major feature of the students' training experience.

\section{Dissemination Model}

All Project materials are intended to be useful in training programs at the graduate and postgraduate levels and in program planning. Dissemination is divided into two sections. The first one is active and is aimed at a particular target audience (teacher trainers and stage program planners for emotionally disturbed children). The second one is more passive and is aimed at a broader audience of related professional disciplines. For the active dissemination effort, a plan was developed incorporating a preliminary circulation of papers followed by an intensive three-day workshop, followed by postworkshop written contacts with the various participants. A small group of regional leaders from across the nation comprised the first workshop. This group then became the local leaders and facilitators of a national series of regional workshops. 


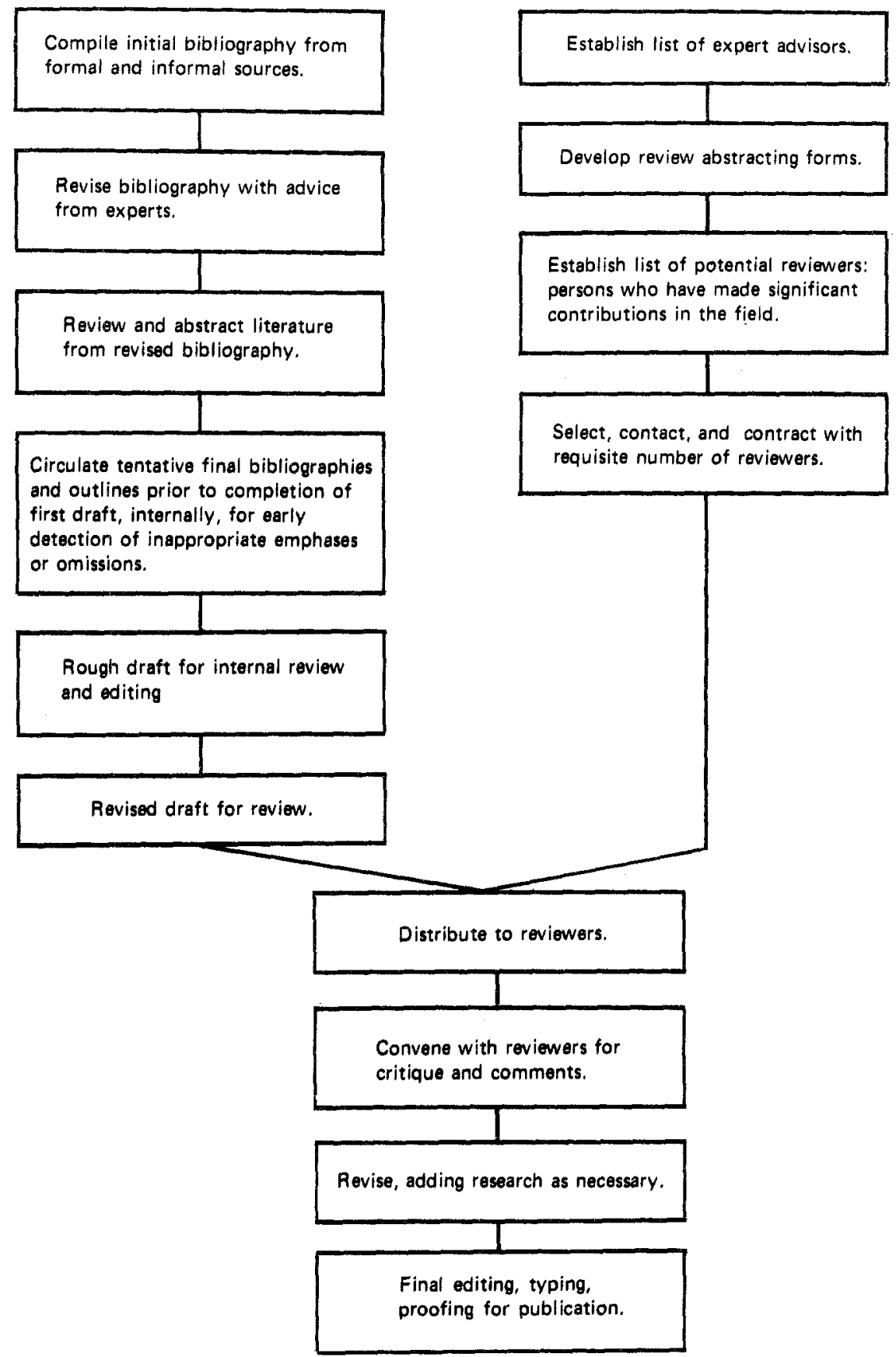

Fig. 2. Authenticated review process model. 
The workshops themselves were developed on a modular basis to allow the workshop model to be self-evolving from one to the next. The model focuses on the process through which the participants engage in and relate to the content, rather than on the content per se. Formally, the workshop model has five basic modules: introduction to the workshop, exposure to the materials, synthesis (a didactic presentation with a question-and-answer session), use of the concepts in back-home contexts, and an integrative wrap-up session. The format alternates between large-group activities and small discussion-group or task-group sessions throughout. To implement the self-evolutionary design, feedback comparing the effectiveness of the different modules was collected at the end of each workshop. This feedback focused development on the least successful modules while allowing the most successful modules to be temporarily stabilized. The selfevolving scheme proved a satisfying experience - the module judged worst in the first two workshops was judged best and received many favorable comments from the participants in the last.

\section{CONCLUSION}

If there is a single conclusion that has been unanimously reached by present and former members of the Project staff over the past few years, it is that the monolithic society is a myth and the one-dimensional views of man embodied in various theories are individually inadequate. The societal monolith looks entirely different from different vantage points and not all of it is seen by any. Man - his spirit, his behavior, his culture - looks different from each of the theoretical viewpoints, and none has a monopoly on the truth.

If a single hope has emerged from these studies to date, it is a hope that the monolith and the one-dimensional view will ultimately be abandoned. It is a hope that the various subcultures will acknowledge the cultural pluralism that already exists, and always has existed, and that they will come to treasure their differences. It is a hope that the theoretical schools will likewise admit to the need for the other schools and to treasure their contributions. It is a hope that the various houses of theoreticians, practitioners, and system administrators will eventually commune together, rather than paying each other sporadic formal visits. It is, finally, a hope that those judged variant will receive a more dignified and human kind of care, that they too will have their day in the sun.

\section{SUMMARY OF PROJECT MATERIALS}

Reprinted August 1973

Rhodes, W. C., \& Tracy, M. L. A Study of Child Variance. Vol. 1. Theories. Ann Arbor: University of Michigan, 1972. (625 pp.) 
Contents:

Introductory overview

Biological bases of childhood behavior disorders

A review of learning and behavior theory as it relates to emotional disturbance in children

A limited literature review of theory of the psychodynamic model

Mental illness as social deviance

Ecological theory as a model for constructing a theory of emotional disturbance

Counter theory

An overview: Toward synthesis of models of disturbance

\section{First printing September 1973}

Rhodes, W. C., \& Tracy, M. L. A Study of Child Variance. Vol 2. Interventions. Ann Arbor: University of Michigan, 1973. (695 pp.)

\section{Contents:}

Overview of interventions

Biophysical interventions in emotional disturbance

Behavioral interventions in emotional disturbance

Psychodynamic interventions in emotional disturbance

Environmental interventions in emotional disturbance

Counter-theoretical interventions in emotional disturbance

The cost of printing these volumes has been underwritten, for the purposes of development and testing, by the Bureau of Education of the Handicapped. 3,4

\section{Works in Progress}

Service-Delivery Systems. A comprehensive review of both the history and the current operating structure of the major, publicly owned behavior-regulating institutions in the United States, followed by a case study of their actual operations in a small Midwestern city. Projected availability: by fall 1974 .

Topics are as follows: educational system, mental health system, social services system, legal/correctional system, religious agencies, and counterinstitutions.

The Future of Child Variance. Thoughts are projected on likely conceptual models and treatment modes for variant children in the next decade. Research will cover probable trends, possible alternatives, and desirable outcomes, as predicted by historical patterns, current trends and developments, and emerging needs.

Topics are as follows: Who is to be regarded as "deviant?" What will theories of variance be like? What kinds of treatment technologies will be developed, who will apply them, who will receive them? What will the institutions delivering "care" look like - will they continue to exist at all? What will the counterculture of today contribute to the mainstream culture of the near future?

${ }^{3}$ Each volume is available for a handling and postage fee of $\$ 1.25$ from The University of Michigan, Publications Distribution Service, 615 East University, Ann Arbor, Michigan 48105.

${ }^{4}$ Instructional materials related to these volumes, including videotapes and annotated bibliographies, are available in limited quantities. For further information, write Lynn F. Ellis, The Conceptual Project, New Academic Village, Route 7, North Berry Chapel Road, Franklin, Tennessee 37064. 


\section{NOTES ADDED IN PROOF}

Since the time of writing of this report, one of the books listed as "in progress" has gone to press and is due before September 1974: Rhodes, W. C., and Head, S., A Study of Child Variance, Volume III: Service-Delivery Systems. Ann Arbor: University of Michigan, 1974.

A rental library of video tapes for pre- and postprofessional training purposes has been made available, covering different viewpoints on theories, interventions, and delivery systems. The series contains 31 video tapes and is collectively entitled $A$ Study of Child Variance.

Additional items have been added to the works in progress: an Exercise Book of classroom materials relating to child variance, containing many research topics, role-play items, discussion exercises, and related materials, with information on a simulated community presented as background; a Data Bank containing a broad spectrum of information and data on the same simulated community for teaching reference purposes is also in preparation. 